for the effusion, which led to the diagnosis of pancreaticopleural fistula with histology confirming that it arose from a pancreatic intraductal papillary mucinous neoplasm.

While pleural effusions are common, those secondary to pancreaticopleural fistulae are rare, most often occurring in patients with a history of chronic pancreatitis or alcoholism [1]. In this case the fistula formed from an IPMN. These are extremely rare entities that represent $10 \%$ of pancreatic cysts. They can show varying degrees of dysplasia with recurrence being rare in noninvasive types $(<8 \%)$ versus invasive types (50-65\%) [2]. Fistulae to abdominal viscera have been described previously [3]; however, to our knowledge, they have not been known to fistulate to the pleural cavity.

Management options are not well defined due to the paucity of cases, but include therapeutic thoracocentesis in conjunction with total parenteral nutrition or somatostatin therapy in an attempt to reduce pancreatic secretions. Interventional approaches involve ERCP with pancreatic duct stenting, pseudocyst drainage or distal pancreatectomy.

Measurement of pleural fluid amylase raised the possibility of a pancreaticopleural fistula in this case. Pleural fluid amylase may be elevated in a number of cases including pancreatic disease, oesophageal rupture or malignancy. A strong association has been shown between amylase-rich effusions and malignancy, most commonly primary lung carcinoma [4]; however, there remains considerable debate over the benefit of measuring pleural fluid amylase in clinical practice. In 2001, BRANCA et al. [5] measured amylase levels in 379 pleural effusions and found that only $1.3 \%$ of cases had an amylase level of $>100 \mathrm{U} \cdot \mathrm{L}^{-1}$. In no case did amylase measurement assist in determining the origin of the effusion [5]. While an association has been shown between amylase-rich effusions and malignancy, only $10-15 \%$ of the malignant effusions in that study were rich in amylase [4]. Currently, the British Thoracic Society guidelines do not recommend routine measurement of pleural fluid amylase [6].

While we do not advocate the routine testing of pleural fluid amylase, this case demonstrates that it may be a worthwhile consideration in cases where the aetiology of an effusion remains unclear.

Our patient remains well and at most recent follow-up, $>18$ months post-surgery, the effusion has not recurred.

\section{Breda Cushen*, Aoife McKeating*, John F. Garvey*,} Jonathan D. Dodd", Hugh Mulcahy ${ }^{\oplus}$, Justin Geoghegan ${ }^{+}$, Edward F. McKone* and Charles G. Gallagher* *Dept of Respiratory Medicine, St. Vincent's University Hospital, "Dept of Radiology, St. Vincent's University Hospital, 'Dept of Gastroenterology, St. Vincent's University Hospital, and ${ }^{+}$Dept of Hepatobiliary Surgery, St. Vincent's University Hospital, Dublin, Ireland.

Correspondence: C.G. Gallagher, National Referral Centre for Adult Cystic Fibrosis, St. Vincent's University Hospital, Elm Park, Dublin 4, Ireland. E-mail: c.gallagher@st-vincents.ie

Statement of Interest: None declared.

\section{REFERENCES}

1 Rockey DC, Cello JP. Pancreaticopleural fistula. Report of 7 patients and review of the literature. Medicine (Baltimore) 1990; 69: 332-344.

2 Bassi C, Sarr MG, Lillemoe KD, et al. Natural history of intraductal papillary mucinous neoplasms (IPMN): current evidence and implications for management. J Gastrointest Surg 2008; 12: 645-650.

3 Shimizu M, Kawaguchi A, Nagao S, et al. A case of intraductal papillary mucinous neoplasm of the pancreas rupturing both the stomach and duodenum. Gastrointest Endosc 2010; 71: 406-412.

4 Villena V, Pérez V, Pozo F, et al. Amylase levels in pleural effusions: a consecutive unselected series of 841 patients. Chest 2002; 121: 470-474.

5 Branca P, Rodriguez RM, Rogers JT, et al. Routine measurement of pleural fluid amylase is not indicated. Arch Intern Med 2001; 161: 228-232.

6 Hooper C, Lee YC, Maskell N, et al. Investigation of a unilateral pleural effusion in adults: British Thoracic Society Pleural Disease Guideline 2010. Thorax 2010; 65: Suppl. 2, ii4-17.

DOI: $10.1183 / 09031936.00035312$

\title{
Lung toxicity in a patient treated with sunitinib
}

\section{To the Editors:}

A 61-yr-old male presented in 2008 with dyspnoea on exertion and night sweats. Diagnosis of mixed connective tissue disease with pulmonary fibrosis was made. The Latex test, Waaler-Rose test and antinuclear antibodies (anti-centromeres) were positive. The computed tomography (CT) image of the abdomen and pelvis was considered normal at that time. He was started on steroids and the dyspnoea improved. Respiratory functional tests remained abnormal with decreased diffusion capacity of the lung for carbon monoxide (DL,CO) (50\%). 6 months later, in April 2009, he developed a fever and macroscopic haematuria. The CT image showed a $9 \mathrm{~cm}$ tumour in the left kidney with latero-aortic lymph nodes and multiple lung metastases (fig. 1a).
There was also evidence of lung fibrosis. A biopsy of the kidney tumour and a lymph node was performed. The results showed evidence of tubulopapillary renal carcinoma (Fürhrman grade II) in the kidney biopsy. The lymph node was involved by a poorly differentiated nonsmall cell carcinoma, different from the kidney lesion. He was referred to the cancer centre. The bone scan and brain magnetic resonance imaging were normal. A left radical nephrectomy with retroperitoneal lymph-node dissection was performed. Pathological examination revealed a $12 \mathrm{~cm}$ high-grade (Führman grade IV) mixed renal-cell carcinoma (clear cell carcinoma, tubulopapillary carcinoma and a sarcomatoid component) with lymph node involvement. It was a pT3a pN2 M1 tumour according to the tumour, node, metastasis 

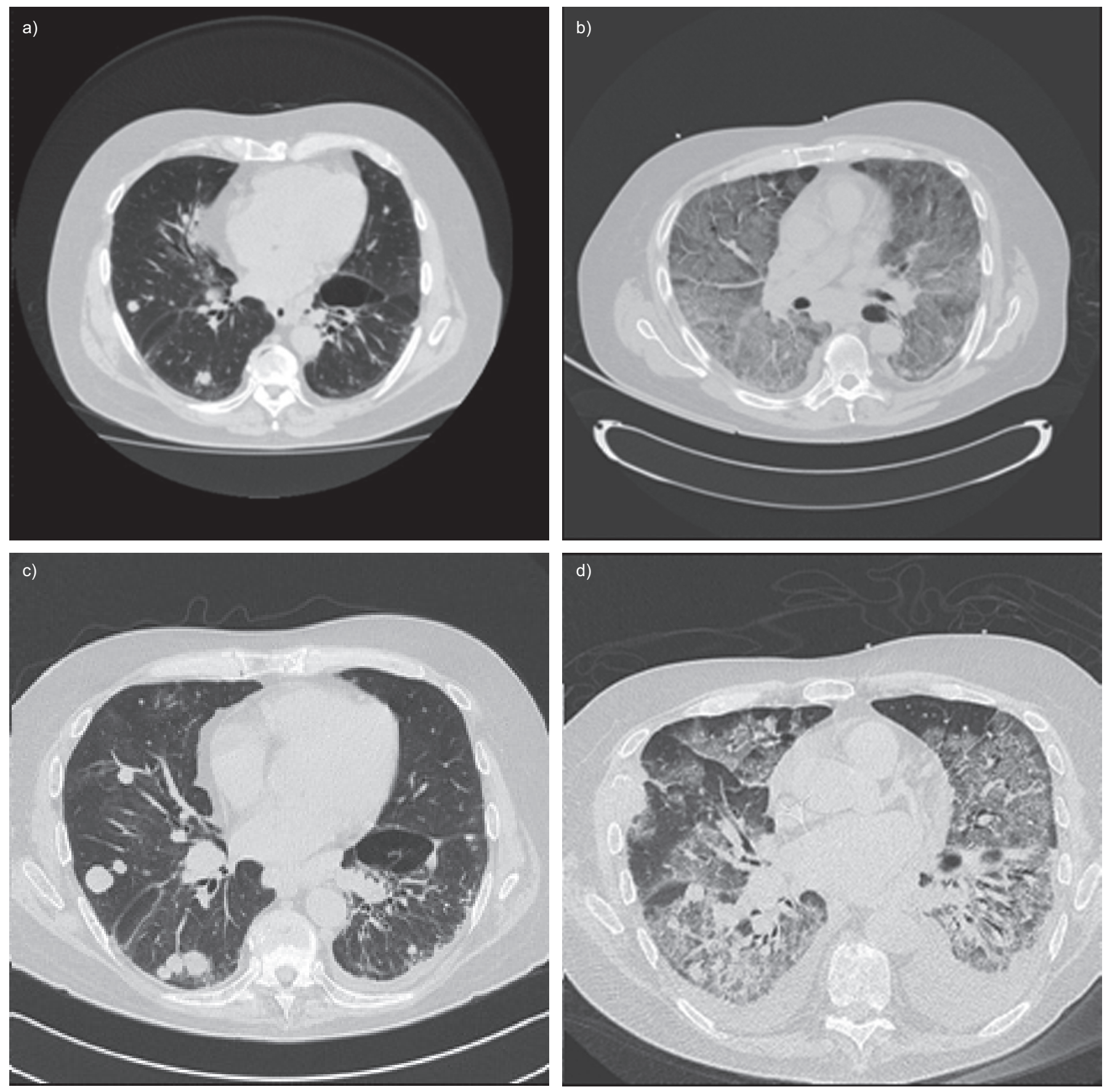

FIGURE 1. Computed tomography images of a 61-yr-old male showing: a) nodules related to metastasis, May 2009; b) diffuse interstitial disease with ground-glass opacities, July 2009; c) progressive lung metastasis, October 2009; and d) diffuse interstitial disease and pleural effusion, October 2009.

(TNM) classification. Postoperative bone scan showed multiple metastases. The patient was started on standard sunitinib treatment $\left(50 \mathrm{mg} \cdot \mathrm{day}^{-1}\right.$ for 4 weeks followed by 2 weeks without treatment) in June 2009.

Initial tolerance of the first cycle of sunitinib was good with grade 1 nausea, dysguesia and skin desquamation, and grade 2 hypertension. Three weeks after starting treatment, he developed pain and fever. He was given ceftriaxone but his general condition worsened and he was admitted into intensive care with breathing difficulties and renal failure. Antibiotics were changed to ciprofloxacin, tazobactam and cotrimoxazole. Despite this treatment the patient worsened and orotracheal intubation was performed. The chest CT image showed bilateral ground-glass opacities (fig. 1b). Infection was ruled out by bronchoalveolar lavage. A surgical lung biopsy was performed. Pathological examination showed acute lesions compatible with cryptogenic organising pneumonia and chronic lesions of interstitial fibrosis. There were no signs of cancer and no infectious agents. The patient was started on steroids and 
clarithromycin. His respiratory status improved quite quickly and he was tailored off the ventilator 6 days after starting steroids. He was then discharged from intensive care. He received clarithromycin at $1,000 \mathrm{mg} \cdot \mathrm{day}^{-1}$ for 10 days and then at $500 \mathrm{mg}$. day $^{-1}$ until the end of week nine, after which he received

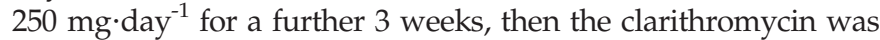
stopped. Prednisone was given at $0.75 \mathrm{mg} \cdot \mathrm{kg}^{-1} \cdot \mathrm{day}^{-1}$ for 2 weeks then at $0.5 \mathrm{mg} \cdot \mathrm{kg}^{-1} \cdot \mathrm{day}^{-1}$ for 2 weeks and then at $20 \mathrm{mg} \cdot \mathrm{day}^{-1}$ for 2 weeks, after which the planned reduction was $10 \mathrm{mg} \cdot \mathrm{day}^{-1}$ for 3 weeks and then $5 \mathrm{mg} \cdot \mathrm{day}^{-1}$ for a further 3 weeks.

The patient progressively improved. Pharmacovigilance declaration was done and a full review of the literature was performed.

When seen in the clinic in October 2009 the CT image showed no signs of pneumonitis but the disease had progressed (lung, liver, lymph node and bone metastases) (fig. 1c). The patient had pain, for which he was on oral prednisolone $\left(40 \mathrm{mg} \cdot \mathrm{day}^{-1}\right)$. He was insistent on being treated. We decided to start him again on sunitinib $25 \mathrm{mg}$ for 3 days and then to stop for 3 days. 4 days after restarting the patient on sunitinib he developed severe dyspnoea. The CT image showed bilateral infiltrates, similar to those seen in July (fig. 1d). Steroids were increased and high-dose furosemide was administered. However, the patient's condition worsened and he died.

Sunitinib is an oral multitargeted inhibitor of: vascular endothelial growth factor receptors-1, -2 , and -3 ; platelet-derived growth factor receptors (PDGFRs) $-\alpha$ and $-\beta$; stem-cell factor receptor (KIT); FMS-like tyrosine kinase 3 (FLT3); colony-stimulating factor-1 receptor; and glial cell line-derived neurotrophic factor receptor (REarranged during Transfection; RET), which is used to treat metastatic kidney cancer.

The most common side-effects of sunitinib are hypertension, diarrhoea, nausea, mucosal inflammation and haematological toxicity. There is only one other report of lung toxicity in the literature [1].

SEIDEL et al. [1] reported on a patient who developed lymphocytic pneumonitis within previously irradiated areas, 6 months after starting sunitinib. Our case is different as our patient had altered respiratory function before starting on sunitinib because of pulmonary fibrosis, the aetiology of which is not clear.

When he progressed, the chest CT image showed no sign of pneumonitis. Because of his altered lung function, mammalian target of rapamycin (mTOR) inhibitors were not a good option and he was too weak to receive interferon plus bevacizumab, therefore, the choice of restarting sunitinib was made.

The chronological order of the events seems to point to a causal effect of sunitinib in this pneumonia. When rechallenged with the drug the symptoms reappeared quickly. Cryptogenic organising pneumonia is defined by intra-alveloar buds of granulation tissue consisting of intermixed myofibroblats and connective tissue. Patients usually present with mild flu-like symptoms: fever, cough and progressively worsening mild dyspnoea. Several aspects can be found on imaging: multiple alveolar opacities, solitary opacity and infiltrative opacities. Patients usually improve dramatically with steroids but can relapse when they are stopped or the dose is reduced. Relapses are usually treated with reintroducing steroids or increasing the doses. Organising pneumonia can be cryptogenic but in some cases a cause can be identified, such as infectious agents, drugs (bleomycin, mTOR inhibitors, etc...), and radiotherapy. It can occur in association with haematological disorders or solid malignancies [2].

Pulmonary toxicity has been described with several targeted therapies.

Severe pneumonitis has been reported with epidermal growth factor receptor inhibitors (erlotinib and gefitinib); cases have also been described with the Her-2 inhibitor trastuzumab. Patients on imatinib, which is a c-kit and a PDGFR inhibitor often present with pleural effusion but some patients develop pneumonitis. Recurrence of pneumonitis has been described after re-exposure to the drug. The PDGF pathway is involved in the regulation of interstitial fluid homeostasis; therefore, it may play a role in this phenomenon [3].

Pneumonitis is a classical side effect of mTOR inhibitors. It has been described in the kidney-transplant population. In the phase III trials of both everolimus and temsirolimus in patients with metastatic kidney cancer, cases of pneumonitis were reported $[4,5]$. In the everolimus trial, pneumonitis occurred in $8 \%$ of patients (any grade); in 3\% of patients it was grade 3 . In the temsirolimus study, $2 \%$ of patients developed pneumonitis (any grade) and for $1 \%$ of patients it was grade $3 / 4$.

The mechanisms by which mTOR inhibitors cause interstitial lung disease are not fully understood $[6,7]$. Several inflammatory cytokines (transforming growth factor- $\beta$, tumour necrosis factor- $\alpha$, PDGF, and interleukins), the nuclear factor$\kappa \beta$ pathway and the production of reactive oxygen species seem to play a role in the development of mTOR inhibitorinduced lung disease. It is also thought that a T-cell delayed hypersensitivity mechanism could be involved.

Risk factors are unknown but in a retrospective study of lung cancer patients treated with everolimus, the presence of interstitial lung disease at baseline was found in $29.2 \%$ of patients who developed pneumonitis and in $7.5 \%$ who did not [8]. This was the case for our patient.

Pneumonitis with sunitinib has rarely been reported. The absence of other identified causes and the positive rechallenge are good arguments for an aetiological link in our case.

\section{Helen J. Boyle*, Gérard Chatté*, Michel Rivoire ${ }^{\#}$ and Aude Fléchon* \\ *Dept of Medical Oncology, Centre Léon Bérard, and "Dept of Surgery, Centre Léon Bérard, Lyon, France.}

Correspondence: H.J. Boyle, Dept of Medical Oncology, Centre Léon Bérard, 28 rue Laennec, 69783 Lyon Cedex 08, France. E-mail: helen.boyle@lyon.unicancer.fr

Statement of Interest: A statement of interest for A. Fléchon can be found at www.erj.ersjournals.com/site/misc/statements.xhtml

\section{REFERENCES}

1 Seidel C, Janssen S, Karstens JH, et al. Recall pneumonitis during systemic treatment with sunitinib. Ann Oncol 2010; 21: 2119-2120.

2 Cordier J-F. Cryptogenic organising pneumonia. Eur Respir J 2006; 28: $422-446$. 
3 Vahid B, Marik PE. Pulmonary complications of novel antineoplastic agents for solid tumors. Chest 2008; 133: 528-538.

4 Bellmunt J, Szczylik C, Feingold J, et al. Temsirolimus safety profile and management of toxic effects in patients with advanced renal cell carcinoma and poor prognostic features. Ann Oncol 2008; 19: 1387-1392.

5 Motzer RJ, Escudier B, Oudard S, et al. RECORD-1 Study Group. Efficacy of everolimus in advanced renal cell carcinoma: a doubleblind, randomised, placebo-controlled phase III trial. Lancet 2008; 372: 449-456.
6 Aparicio G, Calvo MB, Medina V, et al. Comprehensive lung injury pathology induced by mTOR inhibitors. Clin Transl Oncol 2009; 11: 499-510.

7 Duran I, Siu LL, Oza AM, et al. Characterisation of the lung toxicity of the cell cycle inhibitor temsirolimus. Eur J Cancer 2006; 42: 1875-1880.

8 White DA, Schwartz LH, Dimitrijevic S, et al. Characterization of pneumonitis in patients with advanced non-small cell lung cancer treated with everolimus (RAD001). J Thorac Oncol 2009; 4: 1357-1363.

DOI: 10.1183/09031936.00048212 